\title{
A novel biomarker for the detection of esophageal adenocarcinoma
}

\author{
Zane T. Hammoud, MD, ${ }^{a}$ Sunil Badve, MD, ${ }^{\mathrm{b}}$ Romil Saxena, MD, ${ }^{\mathrm{b}}$ Kenneth A. Kesler, MD, ${ }^{\mathrm{a}}$ Karen Rieger, MD, \\ Linda H. Malkas, $\mathrm{PhD}{ }^{\mathrm{c}}$ and Robert J. Hickey, $\mathrm{PhD}^{\mathrm{c}}$
}

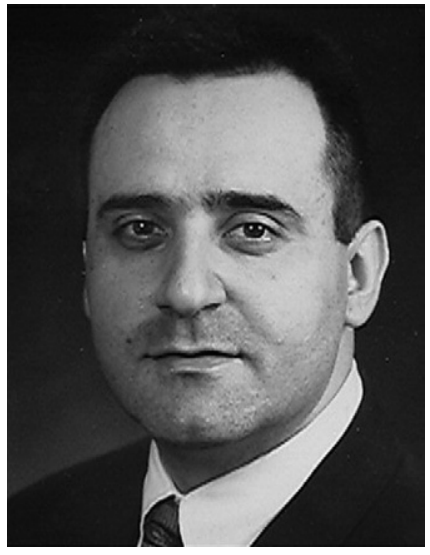

Dr Hammoud
Objectives: Proliferating cell nuclear antigen is a component of the DNA synthesome and functions in DNA replication and repair. Our group has recently identified an acidic isoform of proliferating cell nuclear antigen, cancer-specific proliferating cell nuclear antigen, that appears to be present only in malignant tissue. We sought to determine the presence of cancer-specific proliferating cell nuclear antigen in esophageal dysplasias and invasive adenocarcinomas to assess its potential utility in discriminating malignancy.

Methods: With a polyclonal antibody to cancer-specific proliferating cell nuclear antigen, immunohistochemical staining was performed on samples from a total of 30 patients with Barrett esophagus with varying degrees of dysplasia and 18 patients with invasive adenocarcinoma. We also performed cancer-specific proliferating cell nuclear antigen immunohistochemical staining on a commercially available tissue microarray and on specimens obtained from endoscopic biopsies. As controls, immunohistochemical staining for cancer-specific proliferating cell nuclear antigen was performed on normal esophageal tissue and immunohistochemical staining for proliferating cell nuclear antigen was performed on all specimens with a commercially available antibody.

Results: Of the Barrett esophagus specimen, 14 showed no dysplasia, 8 showed low-grade dysplasia, and 8 showed high-grade dysplasia. None of these specimens stained positively for cancer-specific proliferating cell nuclear antigen. Of the 18 adenocarcinoma specimens, all stained positively for cancer-specific proliferating cell nuclear antigen. There was no significant cancer-specific proliferating cell nuclear antigen expression in normal esophageal tissue, and proliferating cell nuclear antigen expression was noted to a high degree in all tissues.

Conclusions: Cancer-specific proliferating cell nuclear antigen appears to demonstrate high specificity for esophageal adenocarcinoma. This marker therefore may prove useful in differentiating invasive cancer from high-grade dysplasia. Cancerspecific proliferating cell nuclear antigen also holds future promise as a biomarker for esophageal adenocarcinoma.

Read at the Thirty-second Annual Meetin of the Western Thoracic Surgical Association, Sun Valley, Idaho, June 21-24, 2006.

Received for publication June 19, 2006; revisions received Aug 22, 2006; accepted for publication Sept 5, 2006.

Address for reprints: Zane T. Hammoud, MD, 545 Barnhill Dr, EH 215, Indianapolis, IN 46202 (E-mail: zhammoud@iupui.edu).

J Thorac Cardiovasc Surg 2007;133:82-7

$0022-5223 / \$ 32.00$

Copyright (๑) 2007 by The American Association for Thoracic Surgery

doi:10.1016/j.jtcvs.2006.09.011
$\mathrm{D}$ uring the past two decades, the incidence of esophageal adenocarcinoma has increased in many countries, including the United States, at a rate exceeding that of any other malignancy. ${ }^{1}$ Currently, adenocarcinoma of the distal esophagus represents $60 \%$ to $90 \%$ of all esophageal cancers. Esophageal adenocarcinoma, like other malignant processes, develops as a result of a multistep process requiring the accumulation of multiple genetic mutations. This process ultimately results in a cell that is independent of regulated proliferative and cell death pathways and that has the capacity to become invasive and metastasize. Although the precise mechanisms are unknown, it is thought that the transformation 


\section{Abbreviations and Acronyms \\ csPCNA = cancer-specific proliferating cell nuclear antigen \\ csPCNAab $=$ polyclonal antibody to cancer-specific proliferating cell nuclear antigen \\ $\mathrm{KLH}=$ keyhole limpet hemacyanin \\ PBS = phosphate-buffered saline solution \\ PCNA $=$ proliferating cell nuclear antigen}

of a normal cell to a malignant one is dependent on an increased level of genetic alteration that leads to genomic instability. $^{2}$

An additional mechanism by which genetic alterations may occur is an error-prone DNA replication process. In the normal cell, DNA replication occurs with great fidelity, whereas DNA replication in malignant cells tends to be error prone. ${ }^{3}$ During replication, one or more of the components of the DNA replication process may become altered and thus lead to genetic mutations in the malignant cell. An increase in the error frequency associated with the DNA synthetic machinery responsible for the elongation of a cell's DNA could result in the accumulation of mutations. Human cell DNA replication requires the coordinated activity of a number of components. It has been demonstrated that this coordinated activity is mediated by a physically and functionally organized multiprotein complex termed the DNA synthesome. ${ }^{4}$ It has also been demonstrated that the synthesome derived from malignant cells mediates errorprone DNA replication. ${ }^{3}$ Thus alterations in the DNA synthesome, leading to error-prone DNA replication, point to a possible mechanism by which a normal cell is transformed into a malignant cell.

Proliferating cell nuclear antigen (PCNA) is a component of the DNA synthesome and functions in DNA replication and repair. ${ }^{2}$ It has been shown that a unique form of PCNA, cancer-specific PCNA (csPCNA), is found in all malignant breast cells but not in nonmalignant breast cells. ${ }^{3}$ Therefore csPCNA may serve as a biomarker of malignancy. A polyclonal antibody that allows the detection of csPCNA by immunohistochemical staining has been developed by our laboratory and designated $\operatorname{cs} P C N A a b$. We sought to determine whether csPCNA might distinguish nonmalignant esophageal tissue from esophageal adenocarcinoma to explore the possibility that csPCNA represents a novel biomarker for the detection of esophageal adenocarcinoma.

\section{Methods}

\section{Preparation of csPCNAab}

Rabbit polyclonal antibodies were prepared by a commercial vendor (Zymed Laboratories Inc, South San Francisco, Calif) to a peptide fragment of PCNA coupled to keyhole limpet hemacyanin (KLH) through four cysteine residues added to the amino-terminal portion of the peptide. A $100-\mu \mathrm{g}$ portion of the KLH conjugated to a peptide fragment of PCNA contained within amino acids 112 through 150 of the protein was resuspended in complete Freund adjuvant and injected subcutaneously into multiple sites in two female New Zealand White rabbits. The rabbits were rested for 1 month before boosting with a second $100-\mu \mathrm{g}$ dose of the KLHcoupled antigen in incomplete adjuvant. Antibody titer to the antigen was determined by enzyme-linked immunosorbent assay approximately 10 to 14 days after immunization, and after an additional 14-day rest period the animals received another boost of KLH-coupled antigen. Twelve days later, $25 \mathrm{~mL}$ antiserum was collected from each rabbit and stored at $-20^{\circ} \mathrm{C}$. The antiserum was dialyzed against two changes of $20-\mathrm{mmol} / \mathrm{L}$ phosphatebuffered saline solution (PBS), $\mathrm{pH} 7.0$, and loaded onto a protein G Sepharose column (Amersham Pharmacia Biotech AB, Uppsala, Sweden) equilibrated in advance with the PBS. The binding capacity of the gel is $19 \mathrm{mg}$ rabbit immunoglobulin $\mathrm{G} / \mathrm{mL}$ packed gel bed. The column was washed with 10 column volumes of PBS and eluted with 10 volumes of $0.1-\mathrm{mol} / \mathrm{L}$ glycine buffer, $\mathrm{pH} 3.0$. Then 1-mL fractions eluting from the column were collected at a flow rate of 1 to $2 \mathrm{~mL} / \mathrm{min}$ into $0.25 \mathrm{~mL} 0.25-\mathrm{mol} / \mathrm{L}$ tris(hydroxymethyl) aminomethane hydrochloride, $\mathrm{pH}$ 8.0. The concentration of protein in fractions containing the protein peak eluting from the column was determined by Bradford assay, and these fractions were combined and dialyzed against PBS containing 10-mmol/L sodium azide before being stored at $4^{\circ} \mathrm{C}$ until their use in various assays.

\section{Immunohistochemical Staining}

Paraffin sections $(4 \mu \mathrm{m})$ taken on charged slides were deparaffinized in xylene (three changes) and hydrated with graded alcohols and distilled water. Antigen retrieval was performed in citrate buffer ( $\mathrm{pH}$ 6.0) in a microwave oven for 10 minutes with subsequent cooling for 20 minutes. This was followed by blocking of endogenous peroxidase activity with Peroxo-block (Zymed). After rinsing slides in PBS, the slides were incubated with the csPCNA antibody (dilution 1:400) for 1 hour. The antigen-antibody reaction was visualized by the avidin-biotin-peroxidase (Picture Plus Kit: HRP/Fab polymer conjugate; Zymed) with diaminobenzidine (DABplus; DAKO Corporation, Carpinteria, Calif) as the chromogen. These were counterstained with hematoxylin (Vector Laboratories, Inc, Burlingame, Calif). These sections were then cleared in alcohol and xylene. The slides were then mounted with Histomount (Zymed) and visualized. Substitution of primary antibody by PBS or isotype control antibody was done for negative control preparations.

Immunohistochemical staining was performed on archived, paraffin-embedded tissue from specimens available at the Indiana University Medical Center. In addition, staining for csPCNA was performed on a commercially available tissue microarray consisting of 60 invasive esophageal adenocarcinoma samples and 3 samples of normal esophageal squamous mucosa (US Biomax, Inc, Ijamsville, Md). Immunohistochemical staining for PCNA was performed with the commercially available PC10 antibody (PCNA Staining Kit; Zymed).

The study was approved by the Indiana University institutional review board. 


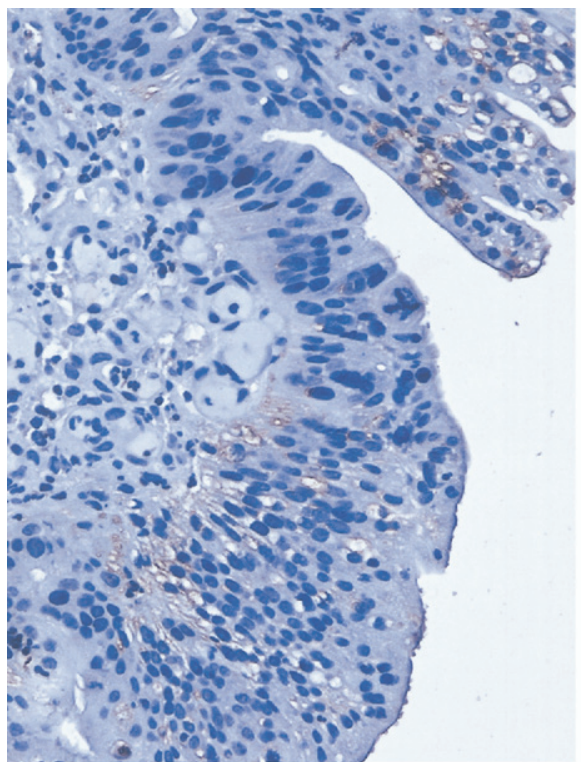

A

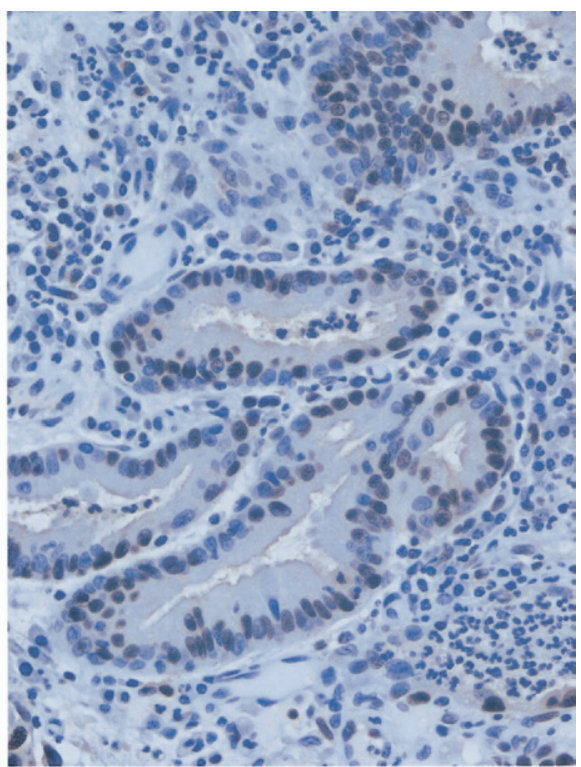

B
Figure 1. Negative staining for cancerspecific proliferating cell nuclear antigen in 2 separate high-grade dysplasia specimens.

\section{Results}

A total of 48 archived, paraffin-embedded samples of abnormal esophageal tissue were examined. These samples came from 14 cases of Barrett esophagus (BE) without histologic evidence of dysplasia, 8 cases of BE with lowgrade dysplasia, 8 cases of BE with high-grade dysplasia, and 18 cases of invasive esophageal adenocarcinoma. Among the $30 \mathrm{BE}$ specimens, none stained positively for csPCNA (Figure 1). Among the 18 adenocarcinoma specimens, all stained positively for csPCNA (Figure 2). All 48 specimens stained positively for PCNA with the commercially available antibody.

In the tissue microarray, all the adenocarcinoma specimens stained positively for csPCNA, whereas there was no significant csPCNA staining of the normal squamous mucosa. Finally, csPCNA staining was performed on various specimens obtained by endoscopic biopsy. All adenocarcinoma specimens stained positively for csPCNA, whereas none of the BE specimens stained positively.

As a control run, 10 specimens of normal esophageal squamous cell mucosa were examined. There was no significant csPCNA staining of these specimens, whereas all stained positively for PCNA (figure 3 ).

\section{Discussion}

PCNA is associated with the human cell DNA synthesome and is required for synthesome-mediated DNA replication in vitro. PCNA is a cofactor for DNA polymerase $\delta$, which is essential for mammalian DNA replication and repair. ${ }^{5}$ It is maximally expressed in the nuclei of late G1-S phase cells and has therefore been used as an indicator of the rate of cell proliferation. High expression of PCNA has been shown to correlate with poor prognosis for several solid tumors. ${ }^{6-8}$ Other studies, however, have failed to demonstrate such a correlation. Bechtel and colleagues ${ }^{9}$ used breast cancer cell lines to demonstrate that PCNA exists in two isoforms, an acidic form and a basic form. They also demonstrated that the acidic form was unique to malignant cells, whereas the basic form could be detected in both malignant and nonmalignant cells. They also showed that the acidic form, now known as csPCNA, was likely the result of an alteration in the posttranslational modification of PCNA, because it was neither proliferation dependent nor the result of a genetic alteration. Further work by our group has led to the isolation of csPCNA and to the development of a polyclonal antibody that can detect this isoform.

With immunohistochemical staining, we have been able to demonstrate that this unique form of PCNA, csPCNA, is detectable in esophageal adenocarcinoma but in neither any of its precursor lesions (BE with varying degrees of dysplasia) nor normal esophageal squamous mucosa. A polyclonal antibody, csPCNAab, successfully detected csPCNA in adenocarcinoma, whereas the basic isoform of PCNA was detected in all tissues with a commercially available antibody. These findings lend further support to previous reports that indicate that csPCNA is detectable only in malignant tissues. Therefore csPCNA may be a marker of malignancy, although much work remains in validating this marker and in assessing its specificity in various tissues.

Our results further suggest that esophageal adenocarcinoma has an altered DNA replication mechanism and that PCNA alterations may play a role in carcinogenesis. The 


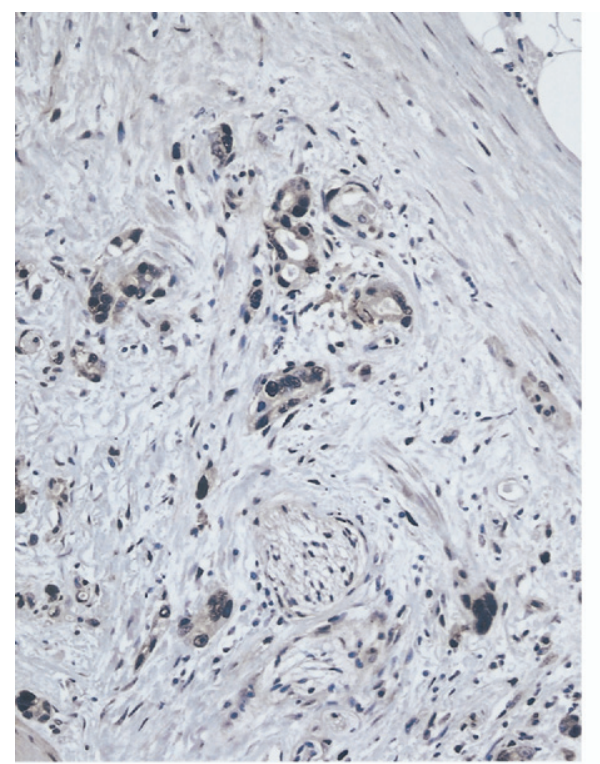

A

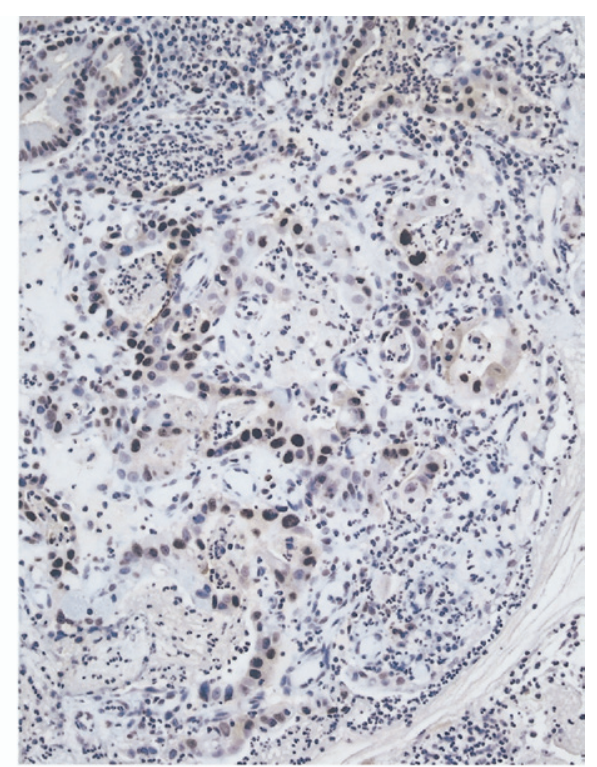

B
Figure 2. Positive staining for cancerspecific proliferating cell nuclear antigen in 2 separate specimens of invasive esophageal adenocarcinoma. detection of csPCNA in esophageal adenocarcinoma may allow earlier detection of this type of malignancy. Currently, histologic evaluation is the only means by which dysplastic changes, most prominently high-grade dysplasia, are differentiated from invasive adenocarcinoma. Although in most cases this is an easy determination, it may sometimes be difficult and subject to pathologic interpretation. By using csPCNA, it may be possible to make such a determination conclusively. When it is difficult to distinguish high-grade dysplasia from invasive adenocarcinoma, positive staining with csPCNA will lend support to the diagnosis of invasive adenocarcinoma, or negative staining will support the diagnosis of high-grade dysplasia only.
Furthermore, it is widely held that as many as $30 \%$ of high grade dysplasias contain foci of invasive cancer; positive staining with csPCNA could help to identify such cases conclusively, potentially sparing patients from invasive procedures designed to treat invasive cancer even in the absence of histologically identifiable cancer on biopsy (esophagectomy).

There are several limitations to our study. Although the detection of csPCNA in esophageal adenocarcinomas by immunohistochemical staining is highly suggestive of alterations in this protein and the DNA replicative machinery, such alterations can only be confirmed by the careful isolation and analysis of the DNA replication apparatus in

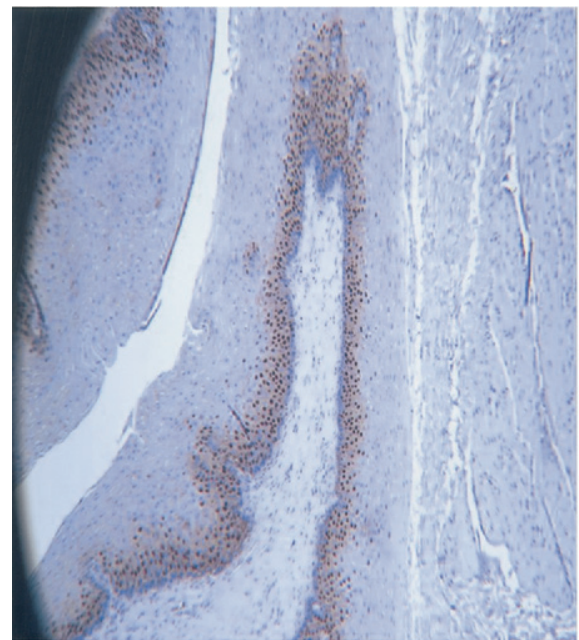

A

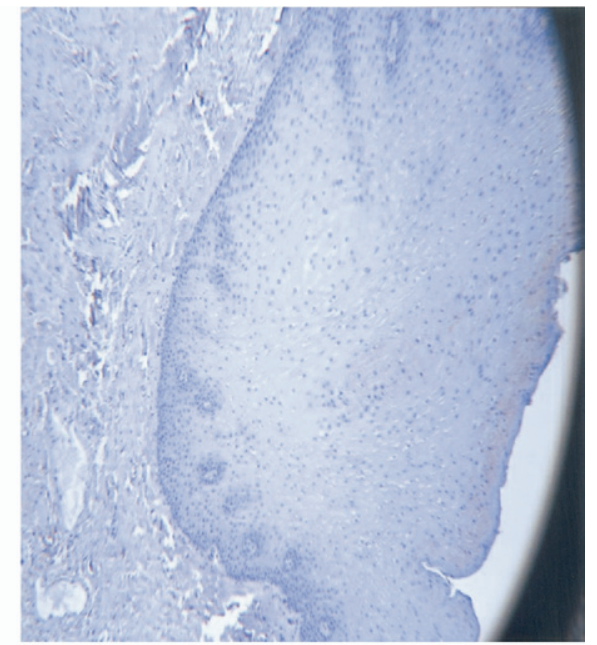

B
Figure 3. Positive staining for proliferating cell nuclear antigen $(A)$ and negative staining for cancer-specific proliferating cell nuclear antigen (B) in normal esophageal squamous mucosa. 
esophageal adenocarcinoma cells. During our immunostaining, we encountered isolated instances of positive csPCNA staining in histologically nonmalignant tissue. The staining pattern in these cases differed slightly between tissue obtained by formal resection and tissue obtained by endoscopic biopsy, indicating a possible effect of tissue preparation on the specificity of csPCNA detection. Although such staining of nonmalignant tissue may indicate nonspecific staining by the csPCNAab, other possibilities include the detection of early malignant changes in histologically nonmalignant-appearing cells as well as an effect of tissue preparation, such as the amount of hypoxic time, on alterations of PCNA. These issues are currently the focus of further investigations into the specificity of the csPCNAab across many tissue types. It should be emphasized, however, that the detection of csPCNA in nonmalignant tissue has been limited, whereas all malignant tissues have stained positively for csPCNA.

Taken together, these preliminary studies suggest that csPCNA may be a marker for esophageal adenocarcinoma. If confirmed by future studies, this finding would have great implications for the treatment of patients with cancer. By using the csPCNAab to follow the status of csPCNA, it may be possible to monitor the response of individual patients to neoadjuvant chemoradiation, with the lack of detection signifying a pathologically complete response. If this technique is proved reliable for the detection of pathologically complete response, it may then be possible to perform a trial comparing the outcomes of patients with pathologically complete response with and without surgery. It may also be possible to diagnose cancer at an early stage, concealed within high-grade dysplasia. The csPCNAab may become a useful member of a panel of antibodies that have the ability to recognize markers currently used to evaluate tumors for their malignant potential, yielding a prognosis. Finally, the csPCNAab may be a useful reagent for developing enzymelinked immunosorbent assays and immunohistochemical assays for screening purposes.

\section{References}

1. Devasa SS, Blot WJ, Fraumeni JF. Changing patterns in the incidence of esophageal and gastric carcinoma in the United States. Cancer. 1998;83:2049-53.

2. Vogelstein B, Kinzler KW. The multistep nature of cancer. Trends Genet. 1993;9:138-41.

3. Sekowski JW, Malkas LH, Schnaper L, Bechtel PE, Long BJ, Hickey RJ. Human breast cancer cells contain an error prone DNA replication apparatus. Cancer Res. 1998;58:3259-63.

4. Hickey RJ, Malkas LH. Mammalian cell DNA replication. Crit Rev Eukaryot Gene Expr. 1997;7:125-57.

5. Malkas LH, Bechtel PE, Sekowski JW, Schnaper L, Lankford CR, Hoelz DJ, et al. A cancer-specific form of proliferating cell nuclear antigen (csPCNA) is present in malignant human breast cells and tissues. J Clin Ligand Assay. 2002;25:20-32.

6. Mayer A, Takimoto M, Fritz E, Schellander G, Kofler K, Ludwig H. The prognostic significance of proliferating cell nuclear antigen, epi- dermal growth factor receptor, and $m d r$ gene expression in colorectal cancer. Cancer. 1993;71:2454-60.

7. Thomas H, Nasim MM, Sarraf CE, Alison MR, Love S, Lambert HE, et al. Proliferating cell nuclear antigen (PCNA) immunostaining-a prognostic factor in ovarian cancer? Br J Cancer. 1995;71:357-62.

8. Jain S, Filipe MI, Hall PA, Waseem N, Lane DP, Levison DA. Prognostic value of proliferating cell nuclear antigen in gastric carcinoma. J Clin Pathol. 1991;44:655-9.

9. Bechtel PE, Hickey RJ, Schnaper L, Sekowski JW, Long BJ, Freund R, et al. A unique form of proliferating cell nuclear antigen is present in malignant breast cells. Cancer Res. 1998;58:3264-9.

\section{Discussion}

Dr Michael Weyant (Denver, Colo). Dr Hammoud, congratulations on your article. I enjoyed reading it, and I appreciate you sending it to me well ahead of the date of this meeting.

You and your coauthors have found a unique biomarker identifying and isolating malignant cells in esophageal tissue. I think findings such as these are important not only because of the ability to mark a cell but also because they can eventually provide some insight into the biology of the cancer cell itself. You also were able to show us a method that is applicable immediately globally to all pathologists, and I think that is important. But I have a few questions for you.

First, given that a pathologist on a routine hematoxylin and eosin stain can identify a cancer cell on the basis of its nuclear morphology, and you have basically to have the presence of the cancer cell to identify it, how is your technique an actual improvement over those already known methods?

Dr Hammoud. I alluded to this earlier. I think a lot of times the differentiation between high-grade dysplasia and invasive carcinoma is difficult and is pathologist dependent. I think the greatest utility right now of having this antibody is in exactly those scenarios. I agree wholeheartedly that you don't need this antibody to diagnose cancer, at least not on a biopsy or a tissue specimen, but I think in those difficult cases it can help. I foresee this as basically being a member of a panel of antibodies that we might be able to use to form some prognostic indicators or, as I said, for those cases where one cannot differentiate high-grade dysplasia from invasive cancer. I think the really exciting potential, however, is the ability to diagnose or pick up csPCNA in the serum. I think that's where the money is, and that's where the biggest clinical impact is potentially going to be.

Dr Weyant. For my second question, and you alluded to this in your article, one of the important applications I see is in looking at groups of patients who have been treated and determining the level of treatment response. We have a group of patients who are determined to have complete response on the basis of pathologic analysis, and they are thought to have a better prognosis. Yet they don't survive forever. I think that with your technique we can stratify groups of these patients. Because these tissues came from your tissue bank at the university, have you had the opportunity to evaluate those patients and their responses to their specific treatments?

Dr Hammoud. The short answer is no; however, we have an Institutional Review Board-approved prospective study underway right now. As at many other institutions, most of our patients, probably $90 \%$, are getting neoadjuvant chemoradiation. Our protocol attempts to predict which patients have a complete pathologic response. At restaging endoscopic ultrasonography after the 
completion of neoadjuvant therapy, if no visible tumor is seen, the endoscopist will perform four quadrant biopsies in the area where the tumor had been seen before treatment. Patients go on to surgical resection, and if in fact they have had a complete pathologic response we can go back and take a look and see whether we could have predicted that complete pathologic response by staining for csPCNA. As you know, $25 \%$ to $30 \%$ of patients will have a complete pathologic response after their neoadjuvant therapy, and the only way to know that is to take the esophagus out. We would like to see whether by using this antibody we can reliably predict which patient has had a complete pathologic response.

Dr Weyant. Great work. Thank you very much.

Dr Hammoud. Thank you.

Dr Donald Low (Seattle, Wash). Once again, I congratulate you on your work. It is potentially another step in the modalities we are all going to be using to determine which patients require what types of treatment. To that end, specifically, one of the issues we're all dealing with right now is that for patients we believe to have true high-grade dysplasia or even intramucosal cancer, we're weighing with risks and benefits of endoscopic versus surgical therapy. One of the things that comes to mind regarding your technique is the ability to increase accuracy of interpretation of the presence or absence of cancer cells up to or through the lamina propria. Have you begun to use this type of technology to increase the acuity of this assessment in presurgical patients, so that we can begin to apply the endoscopic modalities more appropriately?

Dr Hammoud. No, but that is obviously the goal. As I stated in my comments, this is a work in progress. The first goal is for us to try to make a monoclonal antibody. We know from antibodies in general that monoclonals tend to be better than polyclonals. They are certainly easier to work with in terms of developing immunohistochemical conditions, but I agree with you. That is exactly where the clinical applicabilities of this type of antibody lie, and that is where the future is. We also struggle with-and I'm sure a lot of people in this room have similar experiences to us-a lot of the T1 cases not making it to us. They are just being treated, and it's not right, but it happens. It happens in the communities, and it happens at our university even though we beg and plead and tell everybody that these things need to be handled in a true multidisciplinary fashion, but that's the reality. We just talked in the previous discussion about how lung cancers get treated without any of our input. A lot of these T1 and high-grade dysplasia cases are being treated without our input, and needless to say somebody who does that generally doesn't know about this technology or how they can potentially improve patient outcome.

Dr Richard Whyte (Stanford, Calif), Thanks for an interesting presentation. One of the comments you made, or suggested, was that the problem of separating invasive carcinoma from high-grade dysplasia was related to the pathologist reading the slide. I think that this particular issue is more a sampling issue, whereas an interpretative or pathologist-specific issue is distinguishing lowgrade from high-grade dysplasia, where as you know there is a great deal of interobserver variability.

My question to you, though, has to do with the role of this biomarker in either screening patients with Barrett esophagus or discriminating patients with progression from low-grade to highgrade dysplasia or from high-grade dysplasia to invasive adenocarcinoma. Do you have any data on cytologic analysis with this technique?

Dr Hammoud. No. This marker is really present in malignant tissue. The only thing we've shown thus far is its presence in frankly malignant tissue. In both low-grade dysplasia and highgrade dysplasia, none of specimens was stained by this antibody, so it would not be helpful in that regard. Whether we can show differences between high- and low-grade dysplasias is something that I'm working on right now with serum proteomics. I'm looking at normal tissue, low-grade dysplasia, high-grade dysplasia, and cancers and trying to determine protein patterns in serum that might help us spot that progression. 